\title{
MicroRNA expression pattern in pre-eclampsia (Review)
}

\author{
DEEBA S. JAIRAJPURI and WASSIM Y. ALMAWI \\ Department of Medical Biochemistry, Arabian Gulf University, Manama 26671, Kingdom of Bahrain \\ Received May 26, 2015; Accepted January 13, 2016 \\ DOI: $10.3892 / \mathrm{mmr} .2016 .4846$
}

\begin{abstract}
Pre-eclampsia (PE), a pregnancy complication, is a leading cause of maternal and fetal morbidity and mortality. Although its exact etiology and pathogenesis remain elusive, $\mathrm{PE}$ results from an interaction of inherited and non-inherited factors. The clinical symptoms of PE appear post-mid-stage of gestation and, at present, there are no early signs/markers for its onset and progression. MicroRNAs function as gene regulators, and are involved in development and pathology. A burgeoning number of studies have highlighted microRNAs as potential biomarkers for minimal invasive assessment. However, it remains a matter of debate as to which microRNA type is involved in PE onset and progression, as well as the clinical utility of testing for these species. In the present review, we have summarized the latest findings on the association of PE with the aberrant expression of placental microRNAs; in particular, those that are detectable in the blood. The current understanding of the mechanisms of microRNA-target gene interactions that underpin the involvement of microRNAs in the pathogenesis of PE is also discussed.
\end{abstract}

\section{Contents}

1. An overview of PE

2. miRNAs

3. Placenta-specific miRNAs

4. Differential expression of miRNAs in PE

5. Prognostic value of miRNAs in PE

6. Hypothesis bridging miRNAs with PE

7. Conclusion

Correspondence to: Dr Deeba S. Jairajpuri, Department of Medical Biochemistry, Arabian Gulf University, 2904 Road, Manama 26671, Kingdom of Bahrain

E-mail: deebasj@agu.edu.bh

Key words: hypertension, microRNAs, placenta, pre-eclampsia, pregnancy

\section{An overview of pre-eclampsia (PE)}

PE, a pregnancy-associated multisystem disorder, is defined by hypertension, proteinuria and other systemic disturbances in the last trimester of pregnancy, during labor, or shortly following delivery, affecting $\sim 3-8 \%$ of pregnancies worldwide (1). The International Society for the Study of Hypertension in Pregnancy (2) classifies PE as: i) mild PE, with a maternal systolic blood pressure of $\geq 140 \mathrm{mmHg}$ and/or diastolic blood pressure of $\geq 90 \mathrm{mmHg}$ on two occasions separated by $6 \mathrm{~h}$, and significant proteinuria ( $\geq 300 \mathrm{mg}$ protein in a 24 -h urine specimen, or $\geq 1+$ by dipstick) after 20 weeks of gestation; or ii) severe PE, where either severe hypertension (systolic blood pressure of $\geq 160 \mathrm{mmHg}$ and/or diastolic blood pressure of $\geq 110 \mathrm{mmHg}$ on at least two occasions $6 \mathrm{~h}$ apart) plus mild proteinuria or mild hypertension plus severe proteinuria ( $\geq 2 \mathrm{~g} / 24 \mathrm{~h}$, or $\geq 2+$ by dipstick). Several modifiable and non-modifiable factors were documented for increasing the risk of PE. These include first pregnancy, pre-existing diabetes mellitus and insulin resistance, a high body mass index prior to pregnancy, advanced maternal age, renal disease and hypertension (3).

Molecular mechanisms and pathogenesis of PE. PE is termed 'the disease of theories' due to its elusive origin, which is considered to be multifactorial. The clinical manifestations of PE, i.e. the defining lines of the disease, represent the last stage of PE, which begins in the early stages of gestation (4). In general, vascular homeostasis, along with placental and fetal development, is disturbed in PE. The placenta is a key organ involved in pathogenesis, as its removal attenuates the clinical manifestation of PE. Genetic and epigenetic pathways were reported to induce alterations in the placental transcriptome in $\mathrm{PE}$ (5). Observational data have supported the association of PE with endothelial dysfunction, including vasoconstriction and end-organ ischemia (4). For example, circulating and placental levels of soluble fms-like tyrosine kinase-1 (sFlt-1) and soluble endoglin (sEng) are raised in women with PE compared with women experiencing a normal pregnancy (5). sFlt-1 and sEng are anti-angiogenic proteins, which antagonize proangiogenic factors, including vascular endothelial growth factor (VEGF) and placental growth factor, thereby resulting in inadequate placental vascular development (5).

In addition, hypoxia is considered a key determinant of the risk of $\mathrm{PE}$, highlighted by a significant rise in protein levels of hypoxia-inducible factor (HIF) in the placenta of women with PE (6). The restriction of placental perfusion in several animal 
species has resulted in PE-like illness (7), and oxidative stress is central to stimulating the release of cytokines, anti-angiogenic factors and related linkers. Systemic inflammatory responses and endothelial dysfunction in a number of organ systems, including the vasculature, kidney, liver, and so on, was eventually observed in women with PE $(5,6)$. This suggested that a search for a diagnostic, as well as a therapeutic, pathway for $\mathrm{PE}$ requires a thorough assessment of these multiple pathways.

\section{MicroRNAs (miRNAs)}

MiRNAs are regulatory RNAs, 21-23 nucleotides long, which were identified initially in Caenorhabditis elegans in 1993 (8). They are involved in the transcriptional and post-transcriptional regulation of gene expression, and reportedly exert an important role in biological pathways, including cell development, cell differentiation, regulation of cell cycle, metabolism and apoptosis (9). The human genome encodes $>1,000$ miRNA species, and appears to target $60 \%$ of the genes of humans and other mammals (10).

Biogenesis. miRNAs represent a conserved group of nucleotide regulatory RNAs that are involved in multiple pathways (11). miRNAs contribute to the regulatory network by the complementary binding of their 'seed sequences' to targets in the 3'-untranslated region (3'-UTR) of mRNAs (Fig 1). Most miRNAs are involved in regulating the translation of a large number of different mRNAs, with each mRNA possessing multiple binding sites for single or several different miRNAs (12). The most up-to-date comprehensive miRNA database, miRBase, lists 2,578 human mature miRNAs and 1,872 precursors (12).

Role of miRNA in disease. Dysregulation of the expression of miRNAs is associated with several diseases, including diabetes mellitus (13), neurodegeneration, rheumatoid arthritis, gastrointestinal diseases and cancer (9). A burgeoning number of studies have suggested a close link between the disrupted expression of miRNAs and adverse pregnancy complications, including early spontaneous miscarriage (14).

Biochemistry of miRNAs. Tissue- and disease-specific miRNA expression profiles are more discriminatory and stable compared with total mRNA profiles. The biological effectiveness of specific mRNAs resides in their subsequent translation into specific proteins, contrasting with miRNAs, which act as the key regulators of multiple genes; therefore, miRNAs reflect more accurately the altered physiology. Moreover, circulating miRNAs appear to be resistant to endogenous ribonuclease activity, and are therefore fairly stable (15). miRNAs have been demonstrated to be ten times more stable compared with mRNA, the half-life of which is $\sim 10 \mathrm{~h}$ (16). The turnover trend of miRNAs, as determined according to a mathematical model, yielded an average miRNA half-life of $119 \mathrm{~h}$, with certain miRNAs, for example, miR-125b (half-life, $225 \mathrm{~h}$ ), being more persistent compared with others (16). This is likely to be due to their intrinsic structural features, presented in particulate form when associated with exosomes (Fig 1), or in the soluble form when complexed with RNA-binding proteins, such as argonaute 2, or with high-density lipoproteins (17).

Recent advances in polymerase chain reaction (PCR)-based detection methods, such as singleplex real-time quantitative (RT-q)PCR or high-throughput RT-qPCR platforms, has ensured that the quantitative analysis of miRNAs from tissue specimens and plasma/serum has become easier and more precise (Fig. 1) (18). This highlights the ease of exploiting miRNAs as biomarkers, for the development of practicable detection methods for numerous diseases.

Despite the importance of miRNAs as regulators of gene expression, studies which have implicated an altered miRNA expression in obstetrics and gynecology have been limited in scope and number, and the majority of published studies have focused on an association with ovarian (19) or uterine (20) cancers. At present, the link between miRNAs and PE has been addressed by only a few studies. The current review summarizes the state of knowledge pertaining to the role of miRNAs in the pathophysiology of PE, and the possible implications of this association, namely early diagnosis, are discussed.

\section{Placenta-specific miRNAs}

Concerning normal human placenta, the expression of $>600$ miRNAs belonging to the clusters, chromosome 19 miRNA cluster (C19MC), C14MC and miR-371-3, exhibiting gestational-age dependent changes in their expression has been identified (21). A previous report has demonstrated that the placental miRNome predominantly comprises miRNAs belonging to C14MC in the first trimester, and miRNAs from C19MC in the third trimester (22). In particular, C14MC miRNAs are imprinted from the maternal chromosome and C19MC miRNAs are imprinted from the paternal chromosome, both considered to exert important roles in the regulation of cellular differentiation and immunomodulation during pregnancy (22).

C14MC, the largest miRNA cluster comprising 52 miRNA genes, is preserved without major structural changes and is uniquely identified in placental mammals (23). This suggests an important role for C14MC miRNAs in controlling neurogenesis, embryonic development, transcriptional regulation and RNA metabolism (23).

The C19MC cluster, comprising 46 miRNA genes, is implicated for its important role in human embryonic development, similarly to genes such as insulin-like growth factor 2 (24). Notably, several of the miRNAs from the C19MC cluster have been detected in the maternal circulation throughout gestation, including miR-515-3p, miR-516-5p, miR-517a, miR-517c, miR-518b, miR-520a*, miR-520 h, miR-526a and miR-526b (25). Thus, maternal circulating miRNAs may have the potential to be novel diagnostic tools for pregnancy disorders.

The miR-371-3 cluster consists predominantly of three miRNAs, hsa-miR-371-5p, hsa-miR-372 and hsa-miR-373-5p, and is also located on chromosome 19. This cluster is predominantly expressed in the placenta, and the levels of its miRNAs decrease during development (10), and therefore would appear to be essential for cell cycle maintenance and regulation of proliferation and apoptosis. In addition, miRNAs 


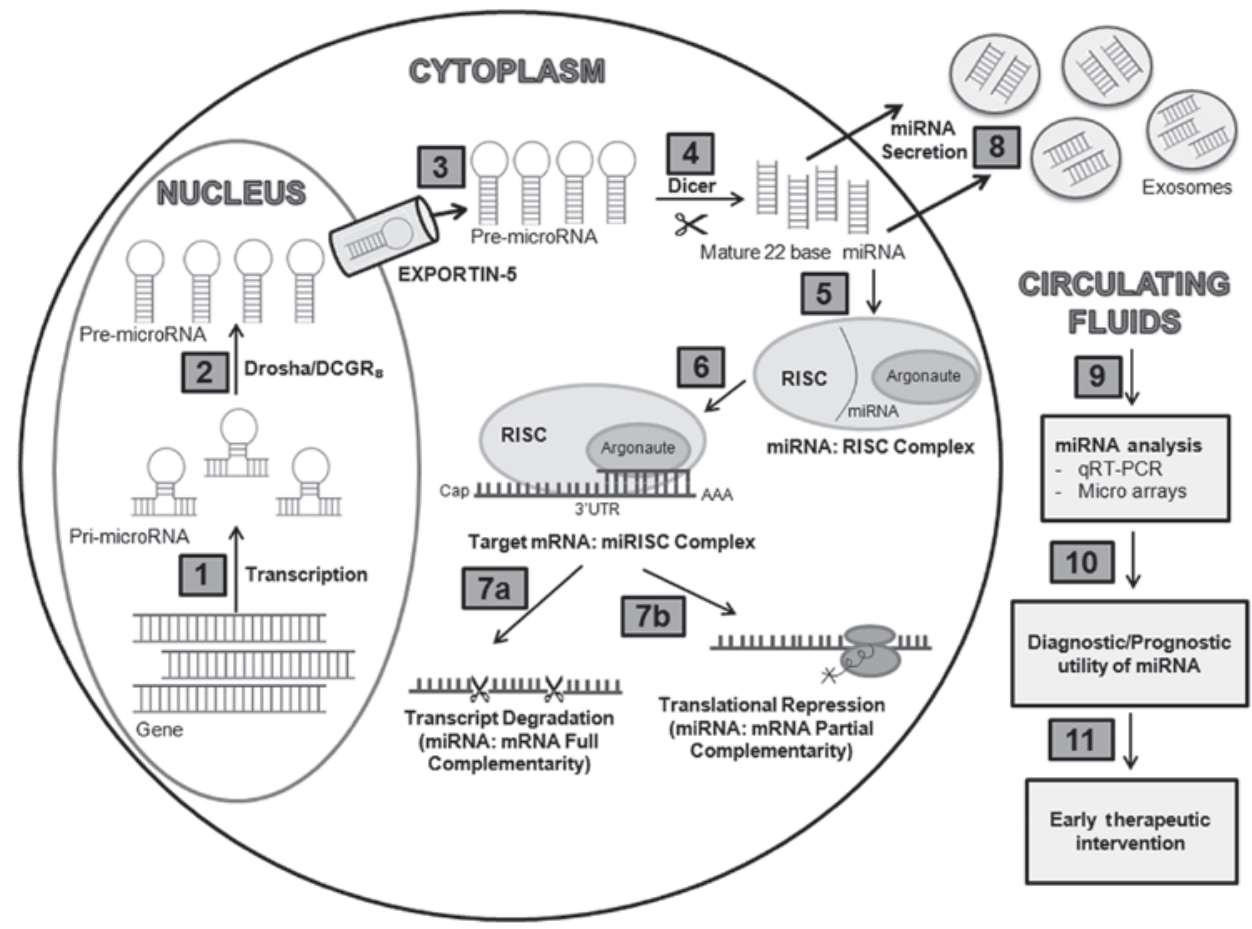

Figure 1. miRNA biogenesis and function. miRNAs are transcribed by RNA polymerase II as Pri-miRNAs (1), which are cleaved by Drosha into Pre-miRNAs almost 70 nucleotides in length (2), prior to being exported from the nucleus into the cytoplasm via an exportin-5/Ran GTP complex (3). Further processing by the RNAse III, Dicer, generates mature miRNA-miRNA duplexes (4). One of the strands of mature miRNA is incorporated into RISC (5), and together they interact with the 3'UTR of the target mRNA (6), leading to either transcript degradation (miRNA: mRNA full complementarity) (7a) or translational repression (miRNA: mRNA partial complementarity) (7b). Mature miRNA duplexes have the tendency to escape cells enclosed in vesicles-exosomes (8), which move to the other cells or are present in the peripheral fluids and can be easily detected by using advanced techniques (9), increasing their scope in diagnosis and allowing early therapeutic intervention $(10,11)$. miRNA, microRNA; qT-PCR, real-time quantitative polymerase chain reaction; RISC, RNA-induced silencing complex; 3'UTR, 3'-untranslated.

from the miR-17-92 cluster have also been observed to be expressed in normotensive term placentas. These miRNAs are important for angiogenesis, affecting the expression of numerous genes, namely HIF1A, interleukin 8, tissue inhibitor of metalloproteinase 2, matrix metallopeptidase 2, VEGFA, ephrin-B and ephrin receptor B4 (21).

The abundant expression of miRNAs (359 miRNAs) in amniotic fluid has been documented, comprising predominantly those involved in maintaining embryonic development and several immunity-related processes, thereby protecting the fetus from foreign pathogens and the maternal host immune response (26).

\section{Differential expression of miRNAs in PE}

Recognition of the importance of miRNAs to the development of PE is a relatively recent phenomenon, dating to 2007, when the first study on the possible association of altered placental miRNA expression with PE was published (27). Subsequent studies have reported detection of miRNA species, predominantly in placental tissues (Table I) (28-32), and more recently, in circulating fluids, such as serum/plasma (Table II) $(30,31,33-35)$. These studies allowed the identification of miRNAs with a minimal overlapping pattern. For example, certain miRNAs, including miR-210, miR-155, miR-196, miR-195, miR-26 and miR-181a, were upregulated $(27,28,30,31,35-37)$, whereas others, including miR-144 and miR-223, were downregulated $(30,33,37)$.
Contradictory data concerning the regulation of other miRNAs in PE were also reported. Furthermore, the upregulation of miR-195 in placentas from severe PE pregnancies (36), as well as downregulation of its expression, were reported $(31,37)$.

It is clear that miRNAs exert an essential role in regulation, as alterations in their expression may result in the dysregulation of several processes, including antiapoptotic survival (27), innate/adaptive immunity $(27,28)$, cell cycle regulation, adhesion and migration (28). This was exemplified by the findings that overexpression of miR-182, miR-182*, miR-155 and miR-210 was associated with increased apoptosis in the placentas of patients with $\mathrm{PE}$, an abnormal immune response and angiogenesis (27). Mice with deficient miRNA exhibited inappropriate angiogenesis, resulting in embryotoxicity (38). In addition, miRNAs may influence signal transduction events (36), vascular remodeling and angiogenesis (21), particularly in cancer metastasis (39), calcium and lipid metabolism (27) and organ/system development (36).

Placental versus circulating miRNAs in PE. Heterogeneity in the sample type, including the placenta (Table I) $(27,32)$, serum $(34,35)$ and plasma (Table II) $(30,31)$, yielded often contradictory findings. This was exemplified by a previous study, which examined miRNA profiles in severe PE in different placenta sites, and in maternal plasma obtained at 15-18 and 35-38 weeks of gestation (31). In placental specimens, a total of seven miRNAs, comprising miR-210, miR-30a-3p, miR-518b, miR-524, miR-17-3p, miR-151 and miR-193b were 
Table I. Differentially expressed miRNAs in pre-eclamptic placentas.

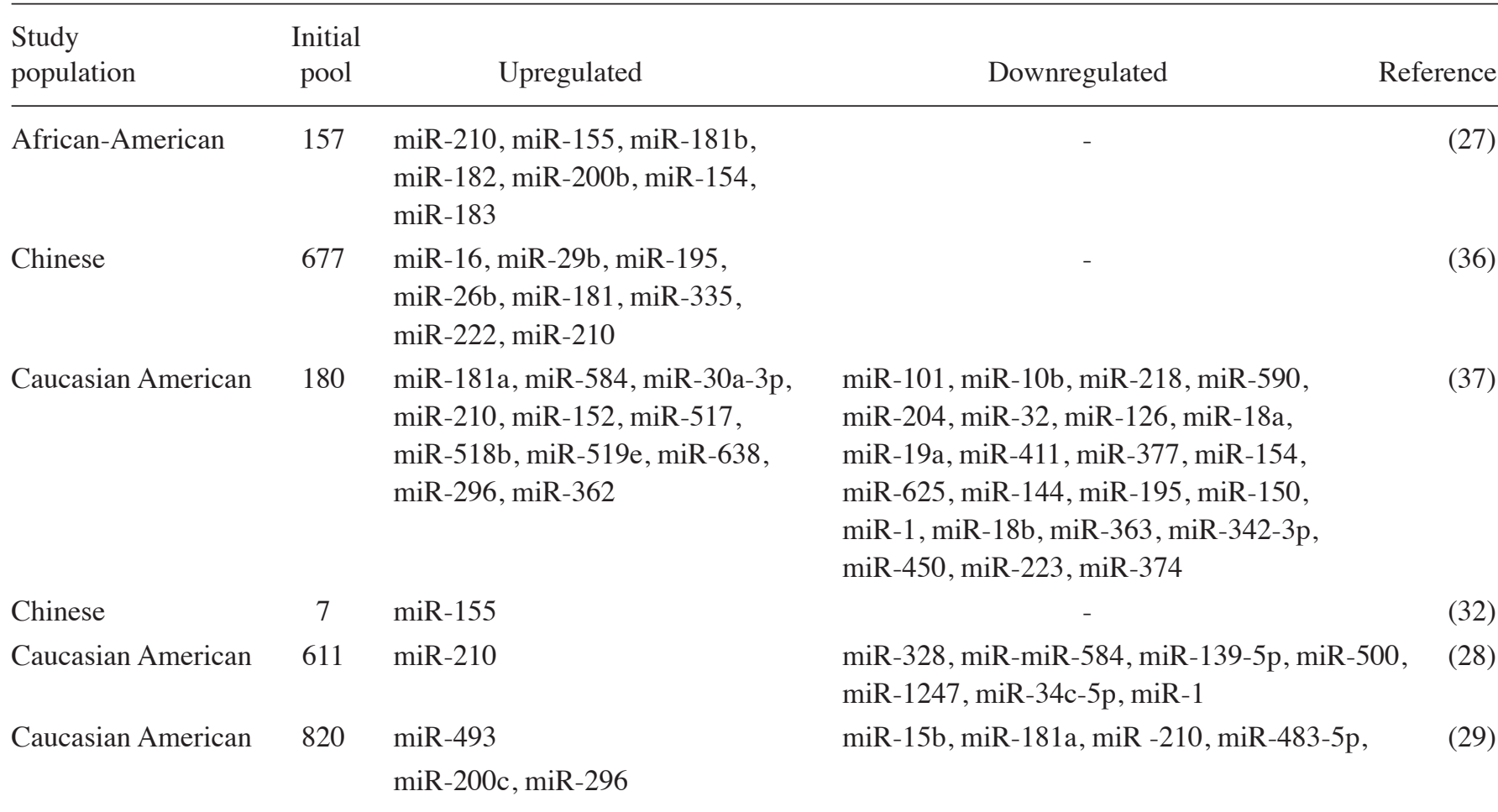

The table lists miRs expressed in plasma/sera, as reported in different studies. miR, microRNA.

upregulated, whereas nine miRNAs, comprising miR-195, miR-223, miR-218, miR-17, miR-18a, miR-196b1, miR-92a1, miR-379 and miR-411, were downregulated (31). However, circulating levels of miR-18a, miR-92a1 and miR-92b1 were markedly lower, whereas that of miR-210 was higher in patients with severe PE at 15-18 gestational weeks, and at term when the plasma was assayed (31).

In addition, differential localization of miRNAs throughout the placenta was reported, as miR-210, miR-518b, miR-524, miR-151 and miR-519e-5p were detected in the basal plate and miR-125, miR-92a1 and miR-379 were expressed in the chorionic plate of placenta (31). This suggests that differences in the sample source may result in differential, and even opposite, results in miRNA profiling (31). The aberrantly expressed miRNAs in this study were linked with modulating trophoblast cell invasion, namely transforming growth factor- 2 signaling via the repression of $\mathrm{Smad} 2 / \mathrm{Smad} 6 / \mathrm{Smad} 7 / \mathrm{Smad} 4 / \mathrm{Smad} 5$ expression, resulting in abnormal placentation.

The placental tissues analyzed in the above-mentioned studies were obtained post-delivery, thereby questioning the exact status of miRNA expression throughout pregnancy. In view of the shortcomings associated with placental sampling, a shift to non-invasive sources of miRNA, including circulating fluids, was suggested (31,33-35).

Role of the severity of PE and onset time. Differential miRNA expression appears to be affected by PE severity, i.e. mild and severe. The differential expression of 51 miRNA species were previously documented, of which 22 were upregulated and five were downregulated in plasma from patients with severe PE, compared with 33 upregulated and six downregulated miRNAs in cases of mild PE (33). Notably, miR-141 and miR-29a were markedly overexpressed in mild PE, whereas miR-144 was underexpressed in mild and severe PE (33). miR-141 was identified as a placenta-specific miRNA (33), miR-144 as a regulator of placental ischemia and hypoxia (40), and miR-29a as a tumor suppressor or promoter (41) in different tumors. In addition, the aberrant expression of miR-29a has previously been observed in diabetes (13) and Alzheimer's disease (42).

The onset time of PE is also an important contributor to miRNA activity. Previous studies reported the dysregulation of 22 (43), 15 (30) and 51 (33) miRNAs in the serum/plasma of women with PE pregnancies in the advanced stage of gestation (third trimester). A more recently published study revealed modest differences in the expression levels of miRNAs in subjects with PE vs. normal pregnancies at the first trimester (34). Of the 754 miRNAs analyzed in pooled sera, 63 were consistently detected in the sera from PE and control subjects, of which 15 miRNAs were differentially expressed with a small fold change (34). Open array profiling confirmed the over-representation of miR-143, miR-125b and miR-192, and the under-representation of miR-126, miR-127, miR-221 and miR-942 in the serum.

Functionally, the miRNAs detected by Luque et al (34) were multipurpose in nature, are were implicated in angiogenesis (miR-125b, miR-143 and miR-942), inflammation (miR-126, miR-127, miR-192 and miR-221) (44), hypoxia/ischemia (miR-127) and cell migration/remodeling (miR-125b, miR-143 and miR-127) (30), rather than being more pregnancy-specific. This suggested a lack of miRNA discriminatory power during the early, preclinical phase of PE, which may be an outcome of the own etiology of the disease, i.e. the appearance 
Table II. Differentially expressed miRNAs in pre-eclamptic circulating fluids.

\begin{tabular}{|c|c|c|c|c|c|}
\hline $\begin{array}{l}\text { Study } \\
\text { population }\end{array}$ & Source & $\begin{array}{c}\text { Initial } \\
\text { pool }\end{array}$ & Upregulated & Downregulated & Reference \\
\hline Chinese & Plasma & 821 & $\begin{array}{l}\text { miR-574-5p, miR-26a, miR-151-3p, } \\
\text { miR-130a, miR-181a, miR-130b, } \\
\text { miR-30d, miR-145, miR-103, miR-425, } \\
\text { miR-221, miR-342-3p, miR-24, miR-210 }\end{array}$ & miR-144, miR-16 & (30) \\
\hline Chinese & Plasma & - & $\begin{array}{l}\text { miR-519d, miR-517b, miR-19a, miR-10a, } \\
\text { miR-517c, miR-18a, miR-210, miR-221, } \\
\text { miR-101, miR-26b, miR-521, miR-378, } \\
\text { miR-519a, miR-520h, miR-125b, miR- } \\
\text { 125a-5p, miR-114, miR-29a, miR-144*, } \\
\text { miR-15b*, miR-182, miR-29c, miR-30a, } \\
\text { miR-518c, miR-27a, miR-24, miR-519e, } \\
\text { miR-130a, miR-515-3p, miR-299a-5p, } \\
\text { miR-518b, miR-23a, miR-23b, miR-34a, } \\
\text { miR-424, miR-525-3p, miR-199a-5p, } \\
\text { miR-100, miR-29b, miR-99a, miR-21, } \\
\text { miR-145, miR-512-5p, miR-30b }\end{array}$ & $\begin{array}{l}\text { miR-19a, miR- } \\
\text { 144, miR-19b, } \\
\text { miR-25, miR-451, } \\
\text { miR-15b, miR-223, } \\
\text { miR-320c, miR-185, } \\
\text { miR-107, Let-7f }\end{array}$ & (33) \\
\hline Chinese & Plasma & 184 & $\operatorname{miR}-210$ & $\begin{array}{l}\operatorname{miR}-18 \mathrm{a}, \mathrm{miR}-19 \mathrm{~b} 1 \\
\text { miR-92a1 }\end{array}$ & (31) \\
\hline $\begin{array}{l}\text { Mixed } \\
\text { population }\end{array}$ & Sera & 63 & miR-143, miR-125b, miR-192 & $\begin{array}{l}\text { miR-126, miR-127, } \\
\text { miR-221, miR-942 }\end{array}$ & (34) \\
\hline Caucasian & Sera & 754 & $\begin{array}{l}\text { miR-1233, miR-650, miR-520a, miR-215, } \\
\text { miR-210, miR-25, miR-518b, } \\
\text { miR-193a-3p, miR-32, miR-204, } \\
\text { miR-296-5p, miR-152 }\end{array}$ & $\begin{array}{l}\operatorname{miR}-126, \operatorname{miR}-335 \\
\text { miR-44, miR-204, } \\
\text { miR-668, miR-376a, } \\
\text { miR-15b }\end{array}$ & $(35)$ \\
\hline
\end{tabular}

The table lists miRs expressed in plasma/sera, as reported in different studies. miR, microRNA.

of miRNAs in the circulation is a relatively late event in PE development (34).

The negative correlation was noted between the levels of miR-942 and maternal arterial pressure, and between the levels of miR-143 and the uterine artery Doppler pulsatility index (34). This was in agreement with an earlier study (30), which documented a role for miRNA-143 in the regulation of blood pressure and vascular function, highlighting the utility of miRNAs as prognostic markers.

Another study along similar lines in Caucasians demonstrated the differential expression of miRNAs in serum samples from an early gestation stage (12-14 weeks) of pregnant women who later developed severe PE in the third trimester (35). A total of 19 mature miRNAs appeared to be differentially expressed, including 12 upregulated (miR-1233, miR-650, miR-520a, miR-215, miR-210, miR-25, miR-518b, miR-193a-3p, miR-32, miR-204, miR-296-5p and miR-152) and seven downregulated (miR-126, miR-335, miR-144, miR-204, miR-668, miR-376a and miR-15b) miRNAs (35). RT-qPCR validated four miRNAs (miR-1233, miR-520a, miR-210 and miR-144), revealing a fold change of $>3$, with miR-1233 exhibiting a fold change of $>5$ between a group of women who developed severe PE compared with a group of women with normotensive pregnancies. Notably, the differentially expressed miRNAs in that study were implicated in different types of cancer, for example, miR-1233 has already been detected in renal carcinoma (45), miR-650 has been implicated in hepatocellular carcinoma (46), and miR-215 and miR-204 have been implicated in metastatic renal cell carcinoma. This clearly suggested a pro-malignancy-like signature of circulating miRNAs in women who develop severe PE at a later stage.

Evidence of placental leakage of miRNAs into the circulation during PE prompted speculation of whether miRNAs could be reliable biomarkers in the timely diagnosis of PE (47). Insofar as the placenta and tumors constitute the primary source of RNA-containing exosomes, the placental miRNAs originating from villous trophoblasts present as placenta-derived exosomes may be detected in the plasma (48). Previously, a research group profiled 377 human miRNAs in placental tissue and the plasma, and 315 of them were observed to be expressed in the placenta, with 286 detectable in the plasma (11).

Effect of ethnic background and technical aspects. Differential expression of miRNAs with a minimum overlap within the studies reported above has been observed, thereby expanding the spectrum of miRNAs implicated in the pathogenesis of 
PE. Such discrepancies can be attributed to the heterogeneity in the ethnic background of study subjects (Tables I and II), as the majority of the reported studies were performed on Chinese populations $(30-33,36)$, whereas the others involved African-American (27), Caucasian American (29), other Caucasian (35) and mixed population (34) subjects.

Variations in the techniques used in miRNA profiling are also considered to be a factor contributing towards inconsistencies in the PE-specific expression of miRNAs. Where certain studies have been performed using RT-qPCR (34), others have utilized high-throughput techniques, such as microarrays (30), and more sophisticated techniques, such as next-generation sequencing (43). A major variability between studies was also observed in statistical methods used for detecting differentially expressed genes. In certain of the studies, an inadequacy in the statistical procedures used was observed, whereas others were not using stringent enough statistical criteria. Whereas certain studies reported only miRNAs with a marked differential expression of a $>2$-fold change $(27,36)$, others exercised a greater leniency, including in their analyses miRNAs with a marked expression of only 1.5 -fold changes $(28,31)$. Other factors which may contribute include the difference in the study sizes, which have been relatively small in the majority of cases, or different definitions of PE used for patient inclusion criteria.

\section{Prognostic value of miRNA in PE}

It is interesting to observe that in PE, thus far, almost 120 miRNAs have been reported to be dysregulated, and none of the two independent studies discussed above have revealed a complete overlapping of the miRNA panel. It is only miR-210 that has been observed in the majority of the studies taken into consideration in the present review, which indicates its importance in normal placentation. miRNA-210 constitutes one of the hypoxia-associated miRNAs ('hypoxamiRs') upregulated by hypoxia (6), and its consistently aberrant expression in $\mathrm{PE}$ makes it a potential serum biomarker for PE (31).

miR-210 has been associated with events integral to the pathogenesis of $\mathrm{PE}$, including the endothelial cell response to hypoxia, formation of capillary-like structures, VEGF-driven cell migration, cell differentiation and survival (28). Upregulation of miR-210 is correlated with the inhibition of migration and the invasive capability of trophoblasts, and is also linked to induction of the activity of several intracellular transcription factors, including nuclear factor- $\mathrm{\kappa B}$ p50 (49). More recently, a study on placentas from healthy pregnant individuals and patients with PE has demonstrated that the aberrant expression of miR-210 may contribute to the occurrence of PE by regulating trophoblast cell invasion via targeting potassium channel modulatory factor-1-mediated signaling in the human placenta (50). Along with miR-210, the aberrant expression of two more miRNAs has been observed in the majority of the studies on PE, and has been suggested to have prognostic value, namely miR-518c (51) and miR-155 (32).

In silico and in vivo studies have demonstrated that the upregulated expression of miR-518c, along with that of miR-210, target and dysregulate the post-transcription of $17-\beta$ hydroxysteroid dehydrogenase 1 (HSD17B1), a placental steriodogenetic enzyme (51). High plasma levels of HSD17B1 observed in women with PE are advantageous, as the enzyme is expected to be derived almost exclusively from the placenta. In vitro studies on BeWo and JEG-3 cells demonstrated upregulation of miR-518c and miR-210 compared with decreased mRNA levels of $H S D 17 B 1$ following exposure to hypoxia, confirming the miRNA-mediated dysregulation of HSD17B1 expression (51).

Similarly, overexpression of miR-155 was reported to downregulate cysteine-rich angiogenic inducer 61 (CYR61), a stress gene expressed by vascular cells and trophoblasts and implicated in cell migration, proliferation, differentiation and adhesion in the placenta of women with PE (32). CYR61 is an important early angiogenic regulating factor during pregnancy, which induces the expression of VEGF, and its 3'-UTR was validated as the target of miR-155 (32). miR-155 appears to control the stability of CYR61 mRNA, and consequently its level of expression thus directly links local ischemia and oxidative stress.

\section{An hypothesis bridging miRNAs with PE}

At present, a large number of studies have documented compelling evidence of aberrantly expressed miRNAs as characteristic phenomena of established PE. A general hypothesis may be drawn that the dysregulated expression of miRNAs observed in PE has a reciprocal effect on their potential target genes, thus curbing or elevating their normal function. Therefore, all the events that occur normally in placental development, as a result of the aberrant expression of miRNAs, now occur abnormally, resulting in impaired cytotrophoblast differentiation and apoptosis, incomplete spiral artery invasion and decreased blood flow to and from the placenta. Trophoblast necrosis releases cell fragments into the maternal bloodstream, which ultimately triggers a systemic immunological response and oxidative stress in the placenta (52).

Furthermore, certain of the miRNAs among the population of dysregulated ones recorded in PE are not only specific for the placenta, but are also part of a number of other organ systems, including the liver, brain, immune system and, most importantly, the kidney. Therefore, any dysregulation of these miRNAs may affect the normal function of target genes in the placenta, as well as in other systems. This explains why $\mathrm{PE}$ is considered to be a multisystem disorder. The stepwise mechanism of action that links miRNAs with PE has yet to be fully elucidated, and thus it is necessary to identify the mechanisms of action for all the miRNAs that account for the occurrence of PE, in order to have a clear and detailed understanding of the pathogenesis of the disease.

\section{Conclusion}

The exact pathogenesis of PE remains incompletely understood. The identification of miRNAs that are reproducibly detected in tissues and circulating fluids suggest their utility as biomarkers. The majority of the differential expression profiles of miRNAs identified in the placenta in cases of PE correlates with those from maternal plasma/sera, demonstrating their prognostic value in PE. However, larger and prospective cohort studies on varied populations are required for the thorough assessment of the contribution of miRNAs to PE. Functional studies, 
including in vitro and in vivo approaches, are warranted to comprehensively elucidate the role of the PE-associated miRNAs in the onset and/or progression of PE. Additionally, it is necessary to delineate the pathways by means of which certain dysregulated miRNAs are able to detrimentally affect a given biological process, and to investigate such pathways with a view to the opportunities they afford for therapeutic intervention in PE.

\section{References}

1. Duley L: The global impact of pre-eclampsia and eclampsia Semin Perinatol 33: 130-137, 2009.

2. ACOG Committee on Practice Bulletin-Obstetrics: ACOG practice bulletin. Diagnosis and management of preeclampsia and eclampsia. Number 33, January 2002. Obstet Gynecol 99: 159-167, 2002.

3. Duckitt K and Harrington D: Risk factors for pre-eclampsia at antenatal booking: Systematic review of controlled studies. BMJ 330: 565-570, 2005.

4. Redman CW and Sargent IL: Latest advances in understanding preeclampsia. Science 308: 1592-1594, 2005.

5. Steegers EA, von Dadelszen P, Duvekot JJ and Pijnenborg R: Pre-eclampsia. Lancet 376: 631-644, 2010.

6. Chan YC, Banerjee J, Choi SY and Sen CK: miR-210: The master hypoxamir. Microcirculation 19: 215-223, 2012.

7. Khalil RA and Granger JP: Vascular mechanisms of increased arterial pressure in preeclampsia: Lessons from animal models. Am J Physiol Regul Integr Comp Physiol 283: R29-R45, 2002.

8. Lee RC, Feinbaum RL and Ambros V: The C. elegans heterochronic gene lin-4 encodes small RNAs with antisense complementarity to lin-14. Cell 75: 843-854, 1993.

9. Jansson MD and Lund AH: MicroRNA and cancer. Mol Oncol 6: 590-610, 2012

10. Bentwich I, Avniel A, Karov Y, Aharonov R, Gilad S, Barad O Barzilai A, Einat P, Einav U, Meiri E, et al: Identification of hundreds of conserved and nonconserved human microRNAs. Nat Genet 37: 766-770, 2005.

11. Devor EC, Santillan DA and Santillan MK: Preeclampsia and MicroRNAs. Proceed Obstet Gynecol 3: 1-10, 2013.

12. Pillar N, Yoffe L, Hod M and Shorman N: The possible involvement of microRNAs in preeclampsia and gestational diabetes mellitus. Best Pract Res Clin Obstet Gynaecol 29: $176-182,2015$

13. Poy MN, Eliasson L, Krutzfeldt J, Kuwajima S, Ma X, Macdonald PE, Pfeffer S, Tuschl T, Rajewsky N, Rorsman P and Stoffel M: A Pancreatic islet-specific microRNA regulates insulin secretion. Nature 432: 226-230, 2004

14. Ventura W, Koide K, Hori K, Yotsumoto J, Sekizawa A, Saito H and Okai T: Placental expression of microRNA-17 and $-19 \mathrm{~b}$ is down-regulated in early pregnancy loss. Eur J Obstet Gynecol Reprod Biol 169: 28-32, 2013.

15. Mitchell PS, Parkin RK, Kroh EM, Fritz BR, Wyman SK, Pogosova-Agadjanyan EL, Pogosova-Agadjanyan EL, Peterson A, Noteboom J, O'Briant KC, et al: Circulating microRNAs as stable blood-based markers for cancer detection. Proc Natl Acad Sci USA 105: 10513-10518, 2008.

16. Gantier MP, McCoy CE, Rusinova I, Saulep D, Wang D, Xu D, Irving AT, Behlke MA, Hertzog PJ, Mackay F and Williams BR Analysis of microRNA turnover in mammalian cells following Dicer1 ablation. Nucleic Acids Res 39: 5692-5703, 2011.

17. Arroyo JD, Chevillet JR, Kroh EM, Ruf IK, Pritchard CC, Gibson DF, Mitchell PS, Bennett CF, Pogosova-Agadjanyan EL, Stirewalt DL, et al: Argonaute2 complexes carry a population of circulating microRNAs independent of vesicles in human plasma. Proc Natl Acad Sci USA 108: 5003-5008, 2011.

18. Kroh EM, Parkin RK, Mitchell PS and Tewari M: Analysis of circulating microRNA biomarkers in plasma and serum using quantitative reverse transcription-PCR (qRT-PCR). Methods 50: 298-301, 2010

19. Yang D, Sun Y, Hu L, Zheng H, Ji P, Pecot CV, Zhao Y, Reynolds S, Cheng H, Rupaimoole R, et al: Integrated analyses identify a master microRNA regulatory network for the mesenchymal subtype in serous ovarian cancer. Cancer Cell 23: 186-199, 2013.
20. Devor EJ,Mik JN, Ramachandran S, Goodheart MJ and Leslie KK: Global dysregulation of the chromosome 14q32 imprinted region in uterine carcinosarcoma. Exp Ther Med 3: 677-682, 2012.

21. Wang W, Feng L, Zhang H, Hachy S, Satohisa S, Laurent LC, Parast M, Zheng J and Chen DB: Preeclampsia up-regulates angiogenesis-associated microRNA (i.e., miR-17,-20a and 20b) that target ephrin-B2 and EPHB4 in human placenta. J Clin Endocrinol Metab 97: 1051-1059, 2012.

22. Morales-Prieto DM, Ospina-Prieto S, Chaiwangyen W, Schoenleben $M$ and Markert UR: Pregnancy-associated miRNA-clusters. J Reprod Immunol 97: 51-61, 2013.

23. Glazov EA, McWilliam S, Barris WC and Dalrymple BP: Origin, evolution and biological role of miRNA cluster in DLK-DIO3 genomic region in placental mammals. Mol Biol Evol 25: 939-948, 2008.

24. Noguer-Dance M, Abu-Amero S, Al-Khtib M, Lefèvre A, Coullin P, Moore GE and Cavaillé J: The primate-specific microRNA gene cluster (C19MC) is imprinted in the placenta. Hum Mol Genet 19: 3566-3582, 2010.

25. Kotlabova K, Doucha J and Hromadnikova I: Placental-specific microRNA in maternal circulation-identification of appropriate pregnancy-associated microRNAs with diagnostic potential. J Reprod Immunol 89: 185-191, 2011.

26. Weber JA, Baxter DH, Zhang S, Huang DY, Huang KH, Lee MJ, Galas DJ and Wang K: The microRNA spectrum in 12 body fluids. Clin Chem 56: 1733-1741, 2010.

27. Pineles BL, Romero R, Montenegro D, Tarca AL, Han YM, Kim YM, Draghici S, Espinoza J, Kusanovic JP, Mittal P, et al: Distinct subsets of microRNAs are expressed differentially in the human placentas of patients with preeclampsia. Am J Obstet Gynecol 196: 261-266, 2007.

28. Enquobahrie DA, Abetew DF, Sorensen TK, Willoughby D, Chidambaram $K$ and Williams MA: Placental microRNA expression in pregnancies complicated by preeclampsia. Am J Obstet Gynecol 204: 12-21, 2011.

29. Mayor-Lynn K, Toloubeydokhti T, Cruz AC and Chegini N: Expression profile of microRNAs and mRNAs in human placentas from pregnancies complicated by preeclampsia and preterm labor. Reprod Sci 18: 46-56, 2011.

30. Wu L, Zhou H, Lin H, Qi J, Zhu C, GaoZ and Wang H: Circulating microRNAs are elevated in plasma from severe preeclamptic pregnancies. Reproduction 143: 389-397, 2012.

31. Xu P, Zhao Y, Liu M, Wang Y, Wang H, Li YX, Zhu X, Yao Y, Wang H, Qiao J, et al: Variations of microRNAs in human placentas and plasma from preeclamptic pregnancy. Hypertension 63: 1276-1284, 2014.

32. Zhang Y, Diao Z, Su L, Sun H, Li R, Cui H and Hu Y: MicroRNA-155 contributes to preeclampsia by down-regulating CYR61. Am J Obstet Gynecol 202: 466-472, 2010.

33. Li H, Ge Q, Guo L and Lu Z: Maternal Plasma miRNAs expression in Preeclamptic Pregnancies. Biomed Res Int 2013: 970265, 2013.

34. Luque A, Farwati A, Crovetto F, Crispi F, Figueras F, Gratacos E and Aran JM: Usefulness of circulating microRNAs for the prediction of early preeclampsia at first-trimester of pregnancy. Sci Rep 4: 4882, 2014

35. Ura B, Feriotto G, Monasta L, Bilel S, Zweyer M and Celeghini C: Potential role of circulating microRNAs as early markers of preeclampsia. Taiwan J Obstet Gynecol 53: 232-234, 2014.

36. Hu Y, Li P, Hao S, Liu L, Zhao J and Hou Y: Differential expression of microRNAs in the placentae of Chinese patients with severe pre-eclampsia. Clin Chem Lab Med 47: 923-929, 2009.

37. Zhu XM, Han T, Sargent IL, Yin GW and Yao YQ: Differential expression profile of microRNAs in human placentas from preeclamptic pregnancies vs. normal pregnancies. Am J Obstet Gynecol 200: 661-667, 2009.

38. Yang WJ, Yang DD, Na S, Sandusky GE, Zhang Q and Zhao G: Dicer is required for embryonic angiogenesis during mouse development. J Biol Chem 280: 9330-9335, 2005.

39. Su X, Chakravarti D, Cho MS, Liu L, Gi YJ, Lin YL, Leung ML, El-Naggar A, Creighton CJ, Suraokar MB, et al: Tap63 suppresses metastasis through coordinate regulation of Dicer and miRNAs. Nature 467: 986-990, 2010

40. Vitoratos N, Hassiakos D and Lavazzo C: Molecular mechanisms of preeclampsia. J Pregnancy 2012: 298343, 2012.

41. Muniyappa MK, Dowling P, Henry M, Meleady P, Doolan P, Gammell P, Clynes M and Barron N: MiRNA-29a regulates the expression of numerous proteins and reduces the invasiveness and proliferation of human carcinoma cell lines. Eur J Cancer 45: 3104-3118, 2009. 
42. Shioya M, Obayashi S, Tabunoki H, Arima K, Saito Y, Ishida T and Satoh J: Aberrant microRNA expression in the brains of neurodegenerative diseases: MiR-29a decreased in Alzheimer disease brains targets neurone navigator 3. Neuropathol Appl Neurobiol 36: 320-330, 2010.

43. Yang Q, Lu J, Wang S, Li H, Ge Q and Lu Z: Application of next-generation sequencing technology to profile the circulating microRNAs in the serum of preeclampsia versus normal pregnant women. Clin Chim Acta 412: 2167-2173, 2011.

44. Xie T, Liang J, Liu N, Wang Q, Li Y, Noble PW and Jiang D: MicroRNA-127 inhibits lung inflammation by targeting IgG Fc $\gamma$ receptor I. J Immunol 188: 2437-2444, 2012.

45. Wulfken LM, Moritz R, Ohlmann C, Holdenrieder S, Jung V, Becker F, Herrmann E, Walgenbach-Brünagel G, von Ruecker A, Müller SC and Ellinger J: MicroRNAs in renal cell carcinoma: Diagnostic implications of serum miR-1233 levels. PLoS One 6: e25787, 2011

46. Zeng ZL, Li FJ, Gao F, Sun DS and Yao L: Upregulation of miR-650 is correlated with down-regulation of ING4 and progression of hepatocellular carcinoma. J Surg Oncol 107: 105-110, 2013.
47. Chim SS, Shing TK, Hung EC, Leung TY, Lau TK, Chiu RW and Lo YM: Detection and characterization of placental microRNAs in maternal plasma. Clin Chem 54: 482-490, 2008.

48. Mincheva-Nilsson L and Baranov V: The role of placental exosomes in reproduction. Am J Reprod Immunol 63: 520-533, 2010.

49. Zhang Y, Fei M, Xue G, Zhou Q, Jia Y, Li L, Xin H and Sun S: Elevated levels of hypoxia-inducible microRNA-210 in pre-eclampsia: New insights into molecular mechanisms for the disease. J Cell Mol Med 16: 249-259, 2012.

50. Luo R, Shao X, Xu P, Liu Y, Wang Y, Zhao Y, Liu M, Ji L, Li YX, Chang C, et al: MicroRNA-210 contributes to preeclampsia by downregulating potassium channel modulatory factor 1 . Hypertension 64: 839-845, 2014

51. Ishibashi O, Ohkuchi A, Ali MM, Kurashina R, Luo S, Ishikawa T, Takizawa T, Hirashima C, Takahashi $\mathrm{K}$, Migita M, et al: Hydroxysteroid (17- $\beta$ ) dehydrogenase 1 is dysregulated by miR-210 and miR-518c that are aberrantly expressed in preeclamptic placentas: A novel marker for predicting preeclampsia. Hypertension 59: 265-273, 2012.

52. Duley L, Henderson-Smart DJ, Meher S and King JF.: Antiplatelet agents for preventing pre-eclampsia and its complications. Cochrane Database Syst Rev. CD004659, 2007. 